\title{
AUTOMATIC CONTINUITY FOR BANACH ALGEBRAS WITH FINITE-DIMENSIONAL RADICAL
}

\author{
HUNG LE PHAM
}

(Received 4 April 2005; revised 21 June 2005)

Communicated by G. Willis

\begin{abstract}
The paper [3] proved a necessary algebraic condition for a Banach algebra $A$ with finite-dimensional radical $R$ to have a unique complete (algebra) norm, and conjectured that this condition is also sufficient. We extend the above theorem. The conjecture is confirmed in the case where $A$ is separable and $A / R$ is commutative, but is shown to fail in general. Similar questions for derivations are discussed.
\end{abstract}

2000 Mathematics subject classification: primary $46 \mathrm{H} 40$.

Keywords and phrases: Banach algebra, uniqueness of norm, automatic continuity, derivation, finitedimensional.

\section{Introduction}

This paper is concerned with the question of uniqueness of norm - the question of when a Banach algebra has a unique complete norm, which means, by definition, that each complete algebra norm on the algebra is equivalent to the given norm. A celebrated theorem of Johnson ([6], [2, Chapter 5.1.6]) states that each semisimple Banach algebra has a unique complete norm. However, it has been known for a long time that even a commutative Banach algebra with 1-dimensional radical may fail to have this property; the first such example is due to Feldman (see [2, Chapter 5.4.6]). There are other conditions that such an algebra needs to satisfy.

The question of uniqueness of norm for algebras with finite-dimensional radical has been discussed in [11], [12], and also implicitly in [4] (see also [2]). The most thorough treatment of this problem has been, however, in [3]. There, the authors propose necessary and sufficient algebraic conditions for a Banach algebra with nonzero, finite-dimensional radical to have a unique complete norm. They proved the

(C) 2006 Australian Mathematical Society $1446-7887 / 06 \$ A 2.00+0.00$ 
following theorem (see [3, Theorem 2.2], or [2, Chapter 5.1.12]). The notation is given in Section 2.

THEOREM. Let A be a unital Banach algebra with finite-dimensional radical. Suppose that there exists a minimal radical ideal $K$ such that $K_{\lambda} K_{\rho}$ has infinite codimension in A. Then A does not have a unique complete norm.

It was conjectured in [3] that the converse of the above theorem holds. This conjecture was proved under various additional hypotheses, but, in its generality, was left unresolved.

In Section 3, we give a new proof of the above theorem. Moreover, we extend the result by proving that the algebra in consideration actually has a discontinuous automorphism. In Section 4, the conjecture is proved in the case where the algebra $A$ is separable and the quotient $A /(\operatorname{rad} A)$ is commutative. This resolves the question, left open in [3], of uniqueness of norm for an interesting class of algebras. Finally, in Section 6, we construct a counter-example to show that the conjecture fails, even in the case where the algebra is separable and the radical is 2-dimensional.

It should be remarked that, for an algebra $A$ with finite-dimensional radical, if $A$ is separable with respect to a complete algebra norm, then $A$ is separable with respect to each complete algebra norm (see [3, Proposition 3.16]).

There is a related question about the automatic continuity of derivations. It is proved in [8] that derivations on each semisimple Banach algebra are continuous. We consider two automatic continuity problems; one is for derivations on Banach algebras with finite-dimensional radical and the other is for derivations from Banach algebras into finite-dimensional Banach modules. Our results on these problems are 'in parallel' to those on the uniqueness-of-norm problem. Moreover, the results on constructing discontinuous automorphisms are all derived from similar results on constructing discontinuous derivations (see Section 3 and Section 6).

There is another related topic, the Wedderburn decomposition of Banach algebras with finite-dimensional radical, which was developed in [7]. However, we do not cover this topic here.

In summary, we prove the following 'characterization' theorems. When $A$ is non-unital we can always consider $A^{*}$ - the unitization of $A$.

THEOREM A. Let A be a separable Banach algebra with finite-dimensional radical $R$ such that $A / R$ is both unital and commutative. Then the following are equivalent:

(a) each derivation on $A$ is continuous;

(b) each derivation $D: A \rightarrow R$ with $D^{2}=0$ is continuous;

(c) each automorphism of $A$ is continuous;

(d) each complete algebra norm on $A$ is equivalent to the given norm; 
(e) for each minimal radical ideal $K$ in $A$, the ideal $K_{\lambda} K_{\rho}$ has finite codimension in $A$.

THEOREM B. Let $A$ be a separable, unital Banach algebra, and let $X$ be a finitedimensional Banach $A$-bimodule such that $A / X^{\perp}$ is commutative. Then the following are equivalent:

(a) each derivation from $A$ into $X$ is continuous;

(b) for each minimal sub-bimodule $Y$ in $X$, the ideal $Y_{\lambda} Y_{\rho}$ has finite codimension in $A$.

\section{Preliminaries}

First, we recall some terminology explained in [3]; see also [2]. Let $A$ be an algebra. For two subspaces $E$ and $F$ in $A$, we denote by $E F$ the linear span of the set $\{a b: a \in E, b \in F\}$.

A minimal radical ideal of $A$ is a minimal non-zero ideal of $A$ that is contained in $\operatorname{rad} A$, the radical of $A$. In each non-zero and finite-dimensional ideal contained in $\operatorname{rad} A$, we can always find a minimal radical ideal.

When $\operatorname{rad} A=R$ is finite-dimensional, there exists $n \in \mathbb{N}$ such that $R^{n}=\{0\}$ and, for each minimal radical ideal $K$, we have $R K=K R=\{0\}$.

For a subspace $I$ of $A$, define the left and right annihilators of $I$ by

$$
I_{\lambda}=\{a \in A: a I=\{0\}\} \text { and } I_{\rho}=\{a \in A: I a=\{0\}\} \text {, }
$$

respectively. Define the annihilator of $I$ by $I^{\perp}=I_{\lambda} \cap I_{\rho}$. Similarly, we can define the left annihilator $X_{\lambda}$ of a left $A$-module $X$ and right annihilator $Y_{\rho}$ of a right $A$-module $Y ; X_{\lambda}$ and $Y_{\rho}$ are ideals in $A$. For an $A$-bimodule $Z$, we also define the annihilator $Z^{\perp}$ to be $Z_{\lambda} \cap Z_{\rho}$.

For each $m \in \mathbb{N}$, let $\mathbb{M}_{m}$ denote the full matrix algebra of $m \times m$-matrices over $\mathbb{C}$. For each $m, n \in \mathbb{N}$, let $\mathbb{M}_{m, n}$ denote the space of $m \times n$-matrices over $\mathbb{C}$; the space $\mathbb{M}_{m, n}$ is naturally considered as an $\mathbb{M}_{m}-\mathbb{M}_{n}$-bimodule.

Let $A$ and $B$ be algebras. Recall that an $A$ - $B$-bimodule $X$ is minimal if $X$ is nonzero and has no other non-zero submodule, and is simple if, furthermore, $A X B \neq 0$. For a finite-dimensional, simple $A$-B-bimodule $X$, there exist $l, r \in \mathbb{N}$ such that $A / X_{\lambda} \cong \mathbb{M}_{l}$ and $B / X_{\rho} \cong \mathbb{M}_{r}$ and, when considered as an $\mathbb{M}_{l}-\mathbb{M}_{r}$-bimodule, $X$ is isomorphic to $\mathbb{M}_{l, r}$; see, for example, [2, page 65]. The module $\mathbb{M}_{l, r}$ is the unique simple $\mathbb{M}_{l}-\mathbb{M}_{r}$-bimodule (up to isomorphism).

Each unital $\mathbb{M}_{l}-\mathbb{M}_{r}$-bimodule can be considered as a unital left $\mathbb{M}_{l r}$-module, and hence each unital $\mathbb{M}_{l}-\mathbb{M}_{r}$-bimodule is semisimple, so that it is an algebraic direct sum of its simple submodules, or equivalently, each of its submodules is an algebraic direct 
summand (in $\mathbb{M}_{l}$-mod- $\mathbb{M}_{r}$ ). For the semisimplicity of unital left $\mathbb{M}_{n}$-modules see, for example, $[9$, pages 28,33$]$.

The following are standard results from automatic continuity theory; for details, see [2]. Let $T: E \rightarrow F$ be a linear operator from a Banach space $E$ into another Banach space $F$. The separating space of $T$, denoted by $\mathfrak{S}(T)$, is defined as

$$
\left\{v \in F \text { : there exists a sequence }\left(u_{n}\right) \subset E \text { with } u_{n} \rightarrow 0 \text { and } T\left(u_{n}\right) \rightarrow v\right\} \text {. }
$$

The space $\mathfrak{S}(T)$ is a closed subspace of $F$ and, by the closed graph theorem, $T$ is continuous if and only if $\mathfrak{S}(T)=\{0\}$. Let $S: F \rightarrow G$ be a bounded linear operator from $F$ into a Banach space $G$. Then we have

$$
\mathfrak{S}(S T)=\overline{S(\mathfrak{S}(T))} ;
$$

see [2, Theorem 5.2.2 (ii)].

Now let $\theta: A \rightarrow B$ be a homomorphism from a Banach algebra $A$ into a Banach algebra $B$. Then the separating space $\mathfrak{S}(\theta)$ is a closed ideal in $\overline{\theta(A)}$. Define the left and right continuity ideals of $\theta$ by

$$
\begin{aligned}
& \mathscr{I}_{\lambda}(\theta)=\{a \in A: b \mapsto \theta(a b), A \rightarrow B, \text { is continuous }\}, \quad \text { and } \\
& \mathscr{I}_{\rho}(\theta)=\{a \in A: b \mapsto \theta(b a), A \rightarrow B, \text { is continuous }\},
\end{aligned}
$$

respectively. By the previous paragraph, we have

$$
\mathscr{I}_{\lambda}(\theta)=\{a \in A: \theta(a) \mathfrak{S}(\theta)=\{0\}\} \quad \text { and } \quad \mathscr{I}_{\rho}(\theta)=\{a \in A: \mathfrak{S}(\theta) \theta(a)=\{0\}\} .
$$

As expected, $\mathscr{I}_{\lambda}(\theta)$ and $\mathscr{I}_{\rho}(\theta)$ are ideals in $A$; in general, they are not closed in $A$.

Let $D$ be a derivation from a Banach algebra $A$ into a Banach $A$-bimodule $X$; by definition, $D$ is a linear map from $A$ into $X$ satisfying

$$
D(a b)=a \cdot D b+(D a) \cdot b \quad(a, b \in A) .
$$

Then the separating space $\mathfrak{S}(D)$ is a closed submodule of $X$. The left and right continuity ideals of $D$ are defined to be

$$
\mathscr{I}_{\lambda}(D)=\{a \in A: a \cdot \mathfrak{S}(D)=\{0\}\} \text { and } \mathscr{I}_{\rho}(D)=\{a \in A: \mathfrak{S}(D) \cdot a=\{0\}\},
$$

respectively. We see that

$$
\begin{aligned}
& \mathscr{I}_{\lambda}(D)=\{a \in A: b \mapsto D(a b), A \rightarrow X, \text { is continuous }\}, \quad \text { and } \\
& \mathscr{I}_{\rho}(D)=\{a \in A: b \mapsto D(b a), A \rightarrow X, \text { is continuous }\}
\end{aligned}
$$

In this case, the ideals $\mathscr{I}_{\lambda}(D)$ and $\mathscr{I}_{\rho}(D)$ are closed in $A$. 


\section{Construction of discontinuous derivations and automorphisms}

In [3, Theorem 2.2], the authors construct directly, on a Banach algebra $A$, a complete algebra norm that is not equivalent to the given norm when $A$ satisfies certain algebraic conditions. Theorem 3.3 below, extends that theorem by providing a discontinuous automorphism of $A$ (as well as a discontinuous derivation on $A$ ). The method is to construct a discontinuous automorphism out of a certain derivation.

First, we construct a discontinuous derivation from a Banach algebra $A$ into a finite-dimensional Banach $A$-bimodule.

LEMMA 3.1. Let $A$ be a Banach algebra, and let $Y$ be a finite-dimensional, simple Banach A-bimodule. Suppose that $A /\left(Y_{\lambda} Y_{\rho}\right)$ is unital and has infinite dimension. Let $F$ be any finite-dimensional subspace of $Y^{\perp}$. Then there exists a discontinuous A-mod-A homomorphism $T$ from $Y^{\perp}$ onto $Y$ such that $T$ is zero on $Y_{\lambda} Y_{\rho}+F$.

PROOF. Since $Y^{\perp}=Y_{\lambda} \cap Y_{\rho}$ has finite codimension in $A$, we see that $Y_{\lambda} Y_{\rho}$ is of infinite codimension in $Y^{\perp}$. Then

$$
I=Y_{\lambda} Y_{\rho}+F+A F+F A+A F A
$$

is also an ideal of infinite codimension in $Y^{\perp}$. Since $A /\left(Y_{\lambda} Y_{\rho}\right)$ is unital, the quotient space $Y^{\perp} / I$ can be considered as a unital $A / Y_{\lambda}-A / Y_{\rho}$-bimodule. As discussed in Section 2, we have that both $A / Y_{\lambda}$ and $A / Y_{\rho}$ are isomorphic to some full matrix algebras, and that $Y^{\perp} / I$ is an algebraic direct sum of simple $A / Y_{\lambda}-A / Y_{\rho}$-submodules. Since $Y^{\perp} / I$ has infinite dimension, there exist distinct such simple submodules, say $E_{n}$ $(n \in \mathbb{N})$. For each $n \in \mathbb{N}$, let $T_{n}$ be an $A / Y_{\lambda}$-mod- $A / Y_{\rho}$ homomorphism from $Y^{\perp} / I$ onto $Y$ such that $T_{n}$ is an isomorphism from $E_{n}$ onto $Y$ and zero on the remaining submodules. Let $\pi: Y^{\perp} \rightarrow Y^{\perp} / I$ be the quotient map. Then each $T_{n} \circ \pi$ is an $A$-mod- $A$ homomorphism onto $Y$, and $T_{n} \circ \pi$ is zero on $I$. It is easily seen that

$$
Y^{\perp}=\bigcup_{n=1}^{\infty} \operatorname{ker}\left(T_{n} \circ \pi\right) .
$$

The Baire category theorem applied to the Banach space $Y^{\perp}$ then shows that $\operatorname{ker}\left(T_{n_{0}} \circ \pi\right)$ is not closed for some $n_{0} \in \mathbb{N}$. Hence, by setting $T=T_{n_{0}} \circ \pi$, we obtain the desired $\operatorname{map} T$.

THEOREM 3.2. Let A be a Banach algebra, and let $X$ be a finite-dimensional Banach A-bimodule such that $A /\left(X_{\lambda} X_{\rho}\right)$ is unital. Suppose that there exists a minimal subbimodule $Y$ of $X$ such that $Y_{\lambda} Y_{\rho}$ has infinite codimension in $A$. Then, for each finitedimensional subspace $F$ of $Y^{\perp}$, there exists a discontinuous derivation $D: A \rightarrow X$ such that $D(A)=Y$ and $D(F)=\{0\}$. 
PROOF. Let $e \in A$ be such that $e+X_{\lambda} X_{\rho}$ is the identity of $A /\left(X_{\lambda} X_{\rho}\right)$.

Since $Y$ is a minimal $A$-bimodule, either $A Y=\{0\}$ or $A Y=Y$. Assume towards a contradiction that $A Y=\{0\}$. Let $a \in Y_{\rho}$. Since $a-e a \in X_{\lambda} X_{\rho}$, we see that

$$
a \in A Y_{\rho}+X_{\lambda} X_{\rho}=Y_{\lambda} Y_{\rho} .
$$

So $Y_{\rho} \subset Y_{\lambda} Y_{\rho}$. This contradicts the assumption that $Y_{\lambda} Y_{\rho}$ has infinite codimension in $A$. Therefore $A Y=Y$ and, similarly, we see that $Y A=Y$. Thus $Y$ is indeed a simple A-bimodule.

Consider the following two cases.

Case 1: $Y_{\lambda}=Y_{\rho}=Y^{\perp}$. Then $A / Y^{\perp} \cong \mathbb{M}_{n}$ for some $n \in \mathbb{N}$. Let

$$
\left\{p_{i j}: i, j=1, \ldots, n\right\} \subset A
$$

be such that $\left\{p_{i j}+Y^{\perp}: i, j=1, \ldots, n\right\}$ corresponds to the standard basis of $\mathbb{M}_{n}$. Then each element $a$ in $A$ can be expressed uniquely as

$$
a=\sum_{i, j \leq n} \alpha_{i j} p_{i j}+u,
$$

where $\alpha_{i j} \in \mathbb{C}$ for $1 \leq i, j \leq n$ and $u \in Y^{\perp}$.

Let $F_{1}$ be the linear span of $F \cup\left\{p_{i j} p_{s t}-\delta_{j s} p_{i t}: 1 \leq i, j, s, t \leq n\right\}$. Then $F_{1}$ is a finite-dimensional subspace of $Y^{\perp}$. Let $T: Y^{\perp} \rightarrow Y$ be a discontinuous $A$-mod- $A$ homomorphism that is zero on $\left(Y^{\perp}\right)^{2}+F_{1}$, the existence of which is guaranteed by Lemma 3.1. Define $D: A \rightarrow Y$ by $D(a)=T(u)$ for each $a \in A$ written in the form specified in (1). It is routine to check that $D$ is a derivation satisfying the requirement. Case 2: $Y_{\lambda} \neq Y_{\rho}$. Since $Y_{\lambda}$ and $Y_{\rho}$ are distinct maximal ideals in $A$ (by the simplicity of $Y$ again), we have $A=Y_{\lambda}+Y_{\rho}$, and so $Y_{\rho} / Y^{\perp} \cong A / Y_{\lambda} \cong \mathbb{M}_{l}$ and $Y_{\lambda} / Y^{\perp} \cong A / Y_{\rho} \cong \mathbb{M}_{r}$, for some $l, r \in \mathbb{N}$. Choose $\left\{p_{i j}: i, j=1, \ldots, l\right\} \subset Y_{\rho}$ and $\left\{q_{i j}: i, j=1, \ldots, r\right\} \subset Y_{\lambda}$ such that

$$
\left\{p_{i j}+Y^{\perp}: i, j=1, \ldots, l\right\} \text { and }\left\{q_{i j}+Y^{\perp}: i, j=1, \ldots, r\right\}
$$

correspond to the standard bases of $\mathbb{M}_{l}$ and $\mathbb{M}_{r}$, respectively. Then we see that each element $a$ in $A$ can be expressed uniquely as

$$
a=\sum_{i, j \leq l} \alpha_{i j} p_{i j}+\sum_{s, t \leq r} \beta_{s t} q_{s t}+u
$$

where $\alpha_{i j} \in \mathbb{C}$ for $1 \leq i, j \leq l, \beta_{s t} \in \mathbb{C}$ for $1 \leq s, t \leq r$, and $u \in Y^{\perp}$.

Let $F_{1}$ be the linear span of

$$
\begin{gathered}
F \cup\left\{p_{i j} q_{s t}: 1 \leq i, j \leq l, \text { and } 1 \leq s, t \leq r\right\} \\
\cup\left\{p_{i j} p_{s t}-\delta_{j s} p_{i t}: 1 \leq i, j, s, t \leq l\right\} \\
\cup\left\{q_{i j} q_{s t}-\delta_{j s} q_{i t}: 1 \leq i, j, s, t \leq r\right\}
\end{gathered}
$$


Then $F_{1}$ is a finite-dimensional subspace of $Y^{\perp}$. By Lemma 3.1, we can find a discontinuous $A$-mod- $A$ homomorphism $T: Y^{\perp} \rightarrow Y$ that is zero on $Y_{\lambda} Y_{\rho}+F_{1}$. Define $D: A \rightarrow Y$ by $D(a)=T(u)$ for each $a \in A$ written in the form specified in (2). Again, it can be verified that $D$ satisfies our requirement.

THEOREM 3.3. Let $A$ be a Banach algebra with finite-dimensional radical $R$ such that $A /\left(R+R_{\lambda} R_{\rho}\right)$ is unital. Suppose that there exists a minimal radical ideal $K$ such that $K_{\lambda} K_{\rho}$ has infinite codimension in $A$. Then:

(1) there exists a discontinuous derivation $D: A \rightarrow R$ with $D^{2}=0$;

(2) there exists a discontinuous automorphism on $A$.

In particular, A has a complete algebra norm which is not equivalent to the given norm.

PROOF. (i) Since $K$ is a minimal radical ideal, we have $R K=K R=\{0\}$. Thus $K$ can be considered as a minimal $A / R$-bimodule. We see that $A / R$ and $K$ satisfy the hypothesis of Theorem 3.2, so there exists a discontinuous derivation $D_{0}: A / R \rightarrow K$. Set $D=D_{0} \circ \pi$, where $\pi: A \rightarrow A / R$ is the quotient map. Then $D: A \rightarrow K$ is a discontinuous derivation with $D(K)=\{0\}$.

(ii) Let $D: A \rightarrow A$ be a discontinuous derivation such that $D^{2}=0$. Denote by $i d_{A}$ the identity map on $A$. Set $\theta=i d_{A}+D$. Then $\theta$ is a discontinuous automorphism on $A$; its inverse map is $\varphi=i d_{A}-D$.

The map $a \mapsto\|\theta(a)\|, A \rightarrow \mathbb{R}^{+}$, is an algebra norm on $A$, easily seen to be complete, and is not equivalent to the given norm.

\section{Banach algebras having a unique complete norm topology}

In this section, we prove the mentioned conjecture for separable Banach algebras with finite-dimensional radical under some additional hypothesis. In particular, we prove the conjecture in the case where $A$ is separable and $A /(\operatorname{rad} A)$ is commutative. This case was proved in [3] under the additional hypothesis that either $\operatorname{rad} A$ is central or $(\operatorname{rad} A)^{2}=\{0\}$. Part of our argument is an extension of the argument in [3]. Our approach is to use separating spaces as well as continuity ideals to prove the continuity of certain homomorphisms.

Let $A$ be an algebra. A composition series of an $A$-bimodule $X$ is a chain $X=$ $X_{0} \supset X_{1} \supset X_{2} \supset \cdots \supset X_{s-1} \supset X_{s}=\{0\}$ of submodules of $X$ such that, for each $1 \leq i \leq s$, the $A$-bimodule $X_{i-1} / X_{i}$ is minimal. We introduce the following concept.

DEFINITION 4.1. An admissible series for an $A$-bimodule $X$ is a chain

$$
X=X_{0} \supset X_{1} \supset X_{2} \supset \cdots \supset X_{s-1} \supset X_{s}=\{0\}
$$


of submodules of $X$ such that the ideals $\left(X_{i-1} / X_{i}\right)_{\lambda}\left(X_{i-1} / X_{i}\right)_{\rho}$, for $1 \leq i \leq s$, are each of finite codimension in $A$.

LEMMA 4.2. Let $A$ be an algebra, and let $X$ be an A-bimodule. Suppose that $X$ has a composition series. Then the following are equivalent:

(a) $X$ has an admissible series;

(b) $X$ has an admissible series that is a composition series;

(c) each composition series of $X$ is an admissible series.

When one of the above three conditions hold then each submodule of $X$ has an admissible series.

Proof. That (a) implies (b) follows by 'refining' the given admissible series to obtain a composition series, which is easily seen to be admissible. That (b) implies (c) follows from the Jordan-Hölder theorem [1, pages 117-120]. The remaining implication ((c) implies (a)) is obvious. The final assertion then follows from (c).

A finite-dimensional $A$-bimodule $X$ always has a composition series.

LEMMA 4.3. Let $A$ and $B$ be commutative algebras, and let $X$ be a finite-dimensional $A$-B-bimodule. Then $X=\bigoplus_{i=1}^{n} X_{i}$ where $X_{i}$ are submodules of $X(1 \leq i \leq n)$ such that, for each $1 \leq i \leq n$, we have

(1) either $A /\left(X_{i}\right)_{\lambda}$ is radical or both $A /\left(X_{i}\right)_{\lambda}$ is local and there exists $a \in A$ such that $a \cdot x=x$ for $x \in X_{i}$, and

(2) either $B /\left(X_{i}\right)_{\rho}$ is radical or both $B /\left(X_{i}\right)_{\rho}$ is local and there exists $b \in B$ such that $x \cdot b=x$ for $x \in X_{i}$.

Proof. Since $X$ is finite-dimensional, we can write $X=\bigoplus_{i=1}^{n} X_{i}$, where $n \in \mathbb{N}$ and each $X_{i}$ is an $A-B$-submodule of $X$ with no non-trivial direct summand (in $A$ $\bmod -B)$. Fix $i$ with $1 \leq i \leq n$. Then $A_{i}=A /\left(X_{i}\right)_{\lambda}$ and $B_{i}=B /\left(X_{i}\right)_{\rho}$ are finite-dimensional, commutative algebras which act faithfully on the left and right of $X_{i}$, respectively. By the Wedderburn structure theorem (see, for example, [2, Chapter 1.5.8]), there exists an orthogonal set $\left\{p_{1}, \ldots, p_{k}\right\}$ (which may be empty) of non-zero idempotents in $A_{i}$ such that

$$
A_{i}=\bigoplus_{j=1}^{k} \mathbb{C} p_{j} \oplus \operatorname{rad} A_{i}
$$

It then follows that

$$
X_{i}=\bigoplus_{j=1}^{k} p_{j} \cdot X_{i} \oplus\left(1-\sum_{j=1}^{k} p_{j}\right) \cdot X_{i}
$$


where $p_{j} \cdot X_{i}(1 \leq j \leq k)$ and $\left(1-\sum_{j=1}^{k} p_{j}\right) \cdot X_{i}$ are easily seen to be $A$ - $B$-bimodules, and each $p_{j} \cdot X_{i}$ is non-zero. Therefore, by the assumption on $X_{i}$, we must have that either $k=0$ or $k=1$. Thus $A_{i}$ is either radical or local; in the later case we must have $p_{1} \cdot x=x(x \in X)$.

The assertion (ii) is proved similarly.

LEMMA 4.4. Let $A$ be an algebra, and let $I_{1}, I_{2}, J_{1}, J_{2}$ be finite codimensional ideals in $A$ such that there exists $m \in \mathbb{N}$ with $J_{1}^{m} \subset I_{1}$ and $J_{2}^{m} \subset I_{2}$. Suppose that $J_{1} J_{2}$ has finite codimension in $A$. Then $I_{1} I_{2}$ has finite codimension in A.

ProOF. This is [3, Lemma 1.6].

Proposition 4.5. Let $A$ be an algebra, and let $X$ be a finite-dimensional $A$ bimodule, such that $A / X^{\perp}$ is commutative. Then the following are equivalent:

(a) for each minimal sub-bimodule $Y$ of $X$, the ideal $Y_{\lambda} Y_{\rho}$ has finite codimension in $A$;

(b) there are sub-bimodules $X_{1}, \ldots, X_{n}$ of $X$ such that $X=\bigoplus_{i=1}^{n} X_{i}$, and such that $\left(X_{i}\right)_{\lambda}\left(X_{i}\right)_{\rho}$ has finite codimension in $A$;

(c) $X$ has an admissible series.

Proof. That (b) implies (c) is obvious, and that (c) implies (a) follows from Lemma 4.2. We prove (a) implies (b).

Suppose that (a) holds. Set $B=A / X^{\perp}$, so that $B$ is a commutative algebra. Let $X=\bigoplus_{i=1}^{n} X_{i}$ be the decomposition as in Lemma 4.3 (working in $B$-mod- $B$ ). Returning to $A$-mod- $A$, we see that the decomposition $X=\bigoplus_{i=1}^{n} X_{i}$ still satisfies the conditions (i) and (ii) in Lemma 4.3.

Fix $1 \leq i \leq n$. Let $Y$ be a minimal sub-bimodule of $X_{i}$. First, if $A /\left(X_{i}\right)_{\lambda}$ is radical, then so is $Y_{\lambda} /\left(X_{i}\right)_{\lambda}$. Otherwise, if $A /\left(X_{i}\right)_{\lambda}$ is local, then, by Lemma 4.3, there exists $a \in A$ such that $a \cdot x=x$ for $x \in X_{i}$, and so $a \notin Y_{\lambda}$. This implies that $Y_{\lambda}$ is a proper ideal in $A$, and therefore, by locality, $Y_{\lambda} /\left(X_{i}\right)_{\lambda}$ is, again, radical. Thus, in both cases, $Y_{\lambda} /\left(X_{i}\right)_{\lambda}$ is radical and finite-dimensional. It follows that $\left(Y_{\lambda}\right)^{l} \subset\left(X_{i}\right)_{\lambda}$ for some $l \in \mathbb{N}$. Similarly, there exists an $r \in \mathbb{N}$ such that $\left(Y_{\rho}\right)^{r} \subset\left(X_{i}\right)_{\rho}$. By hypothesis, we have that $Y_{\lambda} Y_{\rho}$ is of finite codimension in $A$. Hence, by Lemma 4.4, the ideal $\left(X_{i}\right)_{\lambda}\left(X_{i}\right)_{\rho}$ also has finite codimension in $A$.

This completes our proof.

DEFINITION 4.6. Let $A$ be an algebra with a finite-dimensional radical $R$. We say that $A$ satisfies the $A$-property if the $A$-bimodule $R \cap R^{\perp}$ has an admissible series.

That (a) implies (b) from following proposition, in the case where $R^{2}=\{0\}$, was implicit in the proof of [3, Theorem 3.13] (in fact, the argument there can be modified 
to work in the general case of (a) implies (b); our proof is different but still follows the idea of [3]).

PROPOSITION 4.7. Let $A$ be an algebra with finite-dimensional radical $R$ such that $A / R$ is commutative. Then the following are equivalent:

(a) for each minimal radical ideal $K$ in $A$, the ideal $K_{\lambda} K_{\rho}$ has finite codimension in $A$;

(b) there are ideals $R_{1}, \ldots, R_{n}$ in $A$ such that $R \cap R^{\perp}=\bigoplus_{i=1}^{n} R_{i}$, and such that $\left(R_{i}\right)_{\lambda}\left(R_{i}\right)_{\rho}$ has finite codimension in $A$;

(c) A satisfies the A-property.

PROOF. This is a corollary of Proposition 4.5, since each minimal radical ideal $K$ is contained in $R \cap R^{\perp}$.

Lemma 4.8. Let $A$ be a separable Banach algebra, and let $E$ and $F$ be closed linear subspaces of $A$ with $E F$ having finite codimension in $A$. Then $E F$ is closed, and there exist a constant $C>0$ and $m \in \mathbb{N}$ such that, for each $a \in E F$, there exist $\left(x_{i}\right)_{i=1}^{m} \subset E$ and $\left(y_{i}\right)_{i=1}^{m} \subset F$ such that

$$
a=\sum_{i=1}^{m} x_{i} y_{i} \text { and } \sum_{i=1}^{m}\left\|x_{i}\right\|\left\|y_{i}\right\| \leq C\|a\| .
$$

PROOF. This is [3, Lemma 3.1] (see also [2, Chapter 2.2.16] and [10]).

The next result is an extension of Corollaries 3.7-3.9 in [3]. We cannot use an induction scheme (like the one provided by [3, Theorem 3.6]) to prove the next theorem, as was the case for those corollaries.

THEOREM 4.9. Let $A$ be a separable Banach algebra with finite-dimensional radical. Suppose that A satisfies the A-property. Then each complete algebra norm on A is equivalent to the given norm.

PROOF. The radical of $A$ is denoted by $R$. Consider another complete algebra norm $\|\cdot\|$ on $A$. We need to prove that the identity map $\mathfrak{i}:(A,\|\cdot\|) \rightarrow(A,\|\cdot\|)$ is continuous. By Johnson's automatic continuity theorem for epimorphisms, we have $\mathfrak{S}(\mathfrak{i}) \subset R$. For each $a \in R$, since $\mathfrak{i}$ is continuous on $R-$ a finite-dimensional ideal, we have $a \in \mathscr{I}_{\lambda}(\mathfrak{i}) \cap \mathscr{I}_{\rho}(\mathfrak{i})$. Thus, indeed $\mathfrak{S}(\mathfrak{i}) \subset R \cap R^{\perp}$.

Assume towards a contradiction that $\mathfrak{S}(\mathfrak{i}) \neq\{0\}$. Then there exists an ideal $K$ that is maximal among the ideals properly contained in $\mathfrak{S}(\mathfrak{i})$. Since $K$ is finite-dimensional, $K$ is closed in both topologies. Consider the map $\theta:(A,\|\cdot\|) \rightarrow(A / K,\|\cdot\|)$ induced by $i$. Then $\mathfrak{S}(\theta)=\mathfrak{S}(\mathfrak{i}) / K$, and

$$
\mathscr{I}_{\lambda}(\theta)=\{a \in A: \theta(a) \mathfrak{S}(\theta)=\{0\}\}=(\mathfrak{S}(\mathfrak{i}) / K)_{\lambda},
$$


and, similarly, $\mathscr{I}_{\rho}(\theta)=(\mathfrak{S}(i) / K)_{\rho}$. So $\mathscr{I}_{\lambda}(\theta)$ and $\mathscr{I}_{\rho}(\theta)$ are closed (in both topologies). Then, since the bilinear map

$$
T:(x, y) \mapsto \theta(x y), \quad \mathscr{I}_{\lambda}(\theta) \times \mathscr{I}_{\rho}(\theta) \rightarrow A / K
$$

is separately continuous, it follows that $T$ is bounded.

By the $A$-property of $A$ and by Lemma 4.2 , the ideal $\mathscr{I}_{\lambda}(\theta) \mathscr{I}_{\rho}(\theta)$ is of finite codimension in $A$. Then, by Lemma 4.8, we see that $\theta$ is bounded on $\mathscr{I}_{\lambda}(\theta) \mathscr{I}_{\rho}(\theta)$, which is, by the same lemma, a closed, finite-codimensional subspace of $A$. Thus $\theta$ is continuous on the whole of $A$, contradicting the fact that $\mathfrak{S}(\theta)=\mathfrak{S}(\mathfrak{i}) / K \neq\{0\}$. Hence, we must have $\mathfrak{S}(\mathfrak{i})=\{0\}$, implying the continuity of $\mathfrak{i}$.

The following theorem resolves a question that was left open in [3].

THEOREM 4.10. Let $A$ be a separable Banach algebra with finite-dimensional radical $R$ such that $A / R$ is commutative. Suppose that $K_{\lambda} K_{\rho}$ has finite codimension in $A$ for each minimal radical ideal $K$ in $A$. Then each complete algebra norm on $A$ is equivalent to the given norm.

Proof. This follows from Proposition 4.7, and Theorem 4.9.

REMARK. Theorem 4.10 and Proposition 4.7 still hold with the much weaker hypothesis that ' $A / \overline{R+R^{\perp}}$ is commutative' instead of the hypothesis that ' $A / R$ is commutative' (with the same proofs). The algebra $A / \overline{R+R^{\perp}}$ is 'only' finite-dimensional.

\section{The continuity of derivations}

Recall that we are interested in two classes of derivations: derivations on algebras with finite-dimensional radical, and derivations from algebras into finite-dimensional bimodules. The proofs in this section are similar to those in Section 4 .

THEOREM 5.1. Let $D$ be a derivation from a separable Banach algebra A into a Banach A-bimodule $X$. Suppose that $\mathfrak{S}(D)$ is finite-dimensional and has an admissible series as an A-bimodule. Then $D$ is continuous.

Proof. Assume towards a contradiction that $\mathfrak{S}(D) \neq\{0\}$. Then there exists an $A$-bimodule $Y$ which is maximal among the proper $A$-bimodules contained in $\mathfrak{S}(D)$. Consider the map $D_{1}: A \rightarrow X / Y$ induced by $D$. Then $D_{1}$ is a derivation and $\mathfrak{S}\left(D_{1}\right)=\mathfrak{S}(D) / Y$. So $\mathscr{I}_{\lambda}\left(D_{1}\right)=\left\{a \in A: a \cdot \mathfrak{S}\left(D_{1}\right)=\{0\}\right\}=(\mathfrak{S}(D) / Y)_{\lambda}$, and, similarly, $\mathscr{I}_{\rho}\left(D_{1}\right)=(\mathfrak{S}(D) / Y)_{\rho}$.

Continuing as in the proof of Theorem 4.9, we obtain a contradiction. Hence $\mathfrak{S}(D)=\{0\}$, implying the continuity of $D$. 
COROLlARY 5.2. Let $A$ be a separable Banach algebra, and let $X$ be a finitedimensional Banach A-bimodule. Suppose that $X$ has an admissible series as an $A$-bimodule. Then derivations from $A$ into $X$ are continuous.

PROOF. This follows from Proposition 4.2 and Theorem 5.1.

COROLLARY 5.3. Let $A$ be a separable Banach algebra, and let $X$ be a finitedimensional Banach A-bimodule such that $A / X^{\perp}$ is commutative. Suppose that $Y_{\lambda} Y_{\rho}$ has finite codimension in $A$ for each minimal sub-bimodule $Y$ in $X$. Then all derivations from $A$ into $X$ are continuous.

PROOF. This follows from Proposition 4.5 and Corollary 5.2.

REMARK. In [5], a Banach algebra $A$ is constructed whose square $A^{2}$ has finite codimension, but is not closed. Let $X=\mathbb{C}$ with trivial $A$-bimodule actions. Then $A / X^{\perp}=0$. Thus $A$ and $X$ satisfy the hypothesis of Corollary 5.3 except for the separability of $A$. However, any discontinuous linear functional that is zero on $A^{2}$ is a discontinuous derivation from $A$ into $X$. This shows that the separability condition in the hypothesis of the previous corollary and theorem is necessary. We shall see later that the commutativity condition is also necessary (Theorem 6.1). (Un)fortunately, the algebra $A$ is neither commutative nor has finite-dimensional radical. Therefore we cannot say anything about the separability hypothesis in other results.

Now we consider derivations on Banach algebras. It is proved in [8] that, for a semisimple Banach algebra $A$, each derivation $D$ on $A$ is continuous, which means that $\mathfrak{S}(D)=\{0\}=\operatorname{rad} A$. Although it is still open whether $\mathfrak{S}(D) \subset \operatorname{rad} A$ holds for all Banach algebras $A$ and all derivations $D$ on $A$, we have the following result.

LEMMA 5.4. Let $A$ be a Banach algebra with radical $R$ such that $R^{2}$ is closed and has finite codimension in $R$. Then, for each derivation $D$ on $A$, we have $\mathfrak{S}(D) \subset R$.

Proof. We can assume that $A$ is unital with the identity denoted by e. For each primitive ideal $P$ in $A$, define $\pi_{P}$ to be the natural projection from $A$ onto $A / P$.

Assume towards a contradiction that $\mathfrak{S}(D) \not \subset R$. Then $\mathfrak{S}(D) \not \subset P_{1}$ for some primitive ideal $P_{1}$ in $A$, which is equivalent to the discontinuity of $\pi_{P_{1}} D$. By [8, Theorem 3.3], we have $\pi_{P} D$ is continuous for all except finitely many primitive ideals $P_{i}(1 \leq i \leq n)$, and each $P_{i}$ has finite codimension in $A$ (hence is maximal). Now, let $P_{0}$ be the intersection of all the primitive ideals $P$ different from $P_{i}(1 \leq i \leq n)$. Then

$$
P_{0} / R \cong\left(P_{0}+\bigcap_{i=1}^{n} P_{i}\right) / \bigcap_{i=1}^{n} P_{i},
$$


which is finite-dimensional, and so $R^{2}$ is closed and has finite codimension in $P_{0}$. We have $\mathfrak{S}(D) \subset P_{0}$, but $\mathfrak{S}(D) \not \subset P_{1}$, so that $P_{0} \not \subset P_{1}$. Since $P_{1}$ is a maximal ideal in $A$, we see that $P_{0}+P_{1}=A$. In particular, we have $\mathbf{e}=p_{0}+p_{1}$, where $p_{0} \in P_{0}$ and $p_{1} \in P_{1}$. Then $\mathbf{e}=\mathbf{e}^{2}=p+p_{1}^{2}$, where $p=p_{0}^{2}+p_{0} p_{1}+p_{1} p_{0}=p_{0}+p_{1} p_{0} \in P_{0}$. Thus, for each $a \in A$, we have $\pi_{P_{1}} D(a)=\pi_{P_{1}} D(\mathbf{e} a)=\pi_{P_{1}} D(p a)$. This implies that $\pi_{P_{1}} D$ is discontinuous on $P_{0}$. However, it easily seen that $\pi_{P_{1}} D$ is zero on $R^{2}$, a closed subspace of $P_{0}$ of finite codimension, and so $\pi_{P_{1}} D$ is continuous on $P_{0}$, a contradiction.

COROLLARY 5.5. Let A be a separable Banach algebra with finite-dimensional radical. Suppose that A satisfies the A-property. Then derivations on A are continuous.

Proof. The radical of $A$ is denoted by $R$. Let $D: A \rightarrow A$ be a derivation. By Lemma 5.4, we have $\mathfrak{S}(D) \subset R$. As in the proof of Theorem 4.9, we see that $\mathfrak{S}(D) \subset R \cap R^{\perp}$. Hence, the result follows from Theorem 5.1.

COROLLARY 5.6. Let $A$ be a separable Banach algebra with finite-dimensional radical $R$ such that $A / \overline{R+R^{\perp}}$ is commutative. Suppose that $K_{\lambda} K_{\rho}$ has finite codimension in $A$ for each minimal radical ideal $K$ in $A$. Then each derivation on $A$ is continuous.

REMARK. The results in this section hold without modification for a more general class of operators, the intertwining operators. Let $A$ be a Banach algebra. A linear operator $T$ from a Banach $A$-bimodule $X$ into a Banach $A$-bimodule $Y$ is said to be intertwining over $A$ if, for each $a \in A$, the maps

$$
x \mapsto T(a \cdot x)-a \cdot T(x) \quad \text { and } \quad x \mapsto T(x \cdot a)-T(x) \cdot a,
$$

both from $X$ to $Y$, are continuous; see, for instance, [2, Chapter 2.7.1]. Thus each derivation from $A$ into a Banach $A$-bimodule is an intertwining operator.

\section{The general case of dimension at least two}

In this section, we present a counter-example to the main conjecture in [3] mentioned in Section 1.

For a separable Banach algebra $A$ with 1-dimensional radical $R$, the only minimal radical ideal is $R$ itself. We see that the conjecture holds in this case; $A$ has a unique complete norm if (and only if) $R_{\lambda} R_{\rho}$ has finite codimension in $A$, by Theorem 4.9 (and 3.3) or by [3, Corollary 3.9]. However, for greater dimensions, even for dimension 2, the problem becomes more complicated. In [3, Example 5.5], a separable, 
unital Banach algebra with 2-dimensional radical was successfully constructed, which satisfies the hypothesis of the conjecture, but not the hypothesis of any result in [3] (nor does it satisfy the hypothesis of our results); the construction is rather involved. Yet it was proved directly that this algebra has a unique complete norm.

Our example will be a separable, unital Banach algebra with 2-dimensional radical (showing that the conjecture fails for each dimension greater than 1). Following the approach of our previous construction, we first construct similar examples for the problem of derivations from Banach algebras into 2-dimensional Banach modules, and then for the problem of derivations on Banach algebras with 2-dimensional radical.

THEOREM 6.1. There exist a separable, semisimple, unital Banach algebra $A$ and a 2-dimensional, unital Banach A-bimodule $X$ such that both the following hold:

(a) for each minimal sub-bimodule $Y$ in $X$, the ideal $Y_{\lambda} Y_{\rho}$ has finite codimension in $A$;

(b) there exist a discontinuous derivation $D$ from $A$ into $X$.

The following provides a counter-example to the conjecture.

COROLlARY 6.2. There exists a separable, unital Banach algebra $\mathscr{A}$ with 2dimensional radical such that all the following hold:

(a) for each minimal radical ideal $K$, the ideal $K_{\lambda} K_{\rho}$ has finite codimension in $\mathscr{A}$;

(b) there exists a discontinuous derivation on $\mathscr{A}$;

(c) there exists a discontinuous automorphism on $\mathscr{A}$.

In particular, there exists a complete algebra norm on $\mathscr{A}$ that is not equivalent to the given norm.

Proof. Let $A, X$ and $D: A \rightarrow X$ be as in Theorem 6.1. Set $\mathscr{A}=A \oplus X$. Then, with $\ell^{1}$-norm and with product given by

$$
(a \oplus x)(b \oplus y)=a b \oplus(a y+x b) \quad(a, b \in A, x, y \in M),
$$

$\mathscr{A}$ is a separable, unital Banach algebra with $\operatorname{rad} \mathscr{A}=X$. We extend $D$ by linearity to the whole of $\mathscr{A}$ by mapping $X$ to 0 . Then we obtain a discontinuous derivation on $\mathscr{A}$. Define

$$
\theta: a \oplus x \mapsto a \oplus(D a+x), \quad \mathscr{A} \rightarrow \mathscr{A} .
$$

Then it is easily seen that $\theta$ is a discontinuous automorphism on $\mathscr{A}$. The map

$$
a \oplus x \mapsto\|\theta(a \oplus x)\|, \mathscr{A} \rightarrow \mathbb{B}^{+},
$$

gives a complete algebra norm, say $\|\cdot\|$, on $\mathscr{A}$, and $\|\cdot\|$ is not equivalent to the given norm $\|\cdot\|$. Finally, condition (a) follows from Theorem 6.1 (a). 
PROOF OF THEOREM 6.1. Denote by $\mathbb{M}_{2}^{u}$ the algebra of upper triangular $2 \times 2$ matrices (over $\mathbb{C}$ ).

Let $B$ be a separable, commutative, semisimple Banach algebra such that $B^{2}$ has infinite codimension in $B$ (many such examples exist, for example, $\ell^{1}$ with pointwise product). Denote by $\mathbb{M}_{2}(B)$ the Banach algebra of $2 \times 2$-matrices with coefficients in $B$. Then $\mathbb{M}_{2}(B)$ is also semisimple and separable. Set $A=\mathbb{M}_{2}^{u} \oplus \mathbb{M}_{2}(B)$. A generic element of $A$ has the form

$$
\left(\begin{array}{cc}
\alpha & \beta \\
0 & \gamma
\end{array}\right) \oplus\left(\begin{array}{ll}
s & u \\
t & v
\end{array}\right)
$$

where $\alpha, \beta, \gamma \in \mathbb{C}$ and $s, t, u, v \in B$. Then $A$ is an algebra with pointwise addition and with product being the matrix multiplication, so that $\mathbb{M}_{2}^{u}$ and $\mathbb{M}_{2}(B)$ can be naturally identified with subalgebras of $A$. Further,

$$
\mathbb{M}_{2}^{u} \cdot \mathbb{M}_{2}(B) \subset \mathbb{M}_{2}(B) \text { and } \mathbb{M}_{2}(B) \cdot \mathbb{M}_{2}^{u} \subset \mathbb{M}_{2}(B) \text {. }
$$

Giving $A$ the $\ell^{1}$-norm, we obtain a separable, unital Banach algebra (with the identity given by the identity of $\mathbb{M}_{2}^{u}$ ), and that $\mathbb{M}_{2}(B)$ is a closed ideal of $A$.

We see that $A$ has the following two obvious characters:

$$
\varphi:\left(\begin{array}{cc}
\alpha & \beta \\
0 & \gamma
\end{array}\right) \oplus\left(\begin{array}{cc}
s & u \\
t & v
\end{array}\right) \mapsto \alpha \quad \text { and } \quad \psi:\left(\begin{array}{cc}
\alpha & \beta \\
0 & \gamma
\end{array}\right) \oplus\left(\begin{array}{ll}
s & u \\
t & v
\end{array}\right) \mapsto \gamma
$$

We claim that $A$ is semisimple. Indeed, let

$$
a=\left(\begin{array}{ll}
\alpha & \beta \\
0 & \gamma
\end{array}\right) \oplus\left(\begin{array}{ll}
s & u \\
t & v
\end{array}\right) \in \operatorname{rad} A
$$

Then, since $\varphi(a)=\psi(a)=0$, we must have $\alpha=\gamma=0$. We next see that

$$
a\left(\begin{array}{ll}
1 & 0 \\
0 & 0
\end{array}\right)=\left(\begin{array}{ll}
s & 0 \\
t & 0
\end{array}\right) \text { and }\left(\begin{array}{ll}
0 & 0 \\
0 & 1
\end{array}\right) a=\left(\begin{array}{ll}
0 & 0 \\
t & v
\end{array}\right)
$$

so the two elements on the right are in $\mathbb{M}_{2}(B) \cap \operatorname{rad} A=\operatorname{rad} \mathbb{M}_{2}(B)$, and so $s=t=$ $v=0$. Now, let $w \in B$. Multiplying $a$ on the left by $\left(\begin{array}{ll}0 & 0 \\ w & 0\end{array}\right)$, we obtain

$$
\left(\begin{array}{cc}
0 & 0 \\
0 & \beta w+w u
\end{array}\right)
$$

which, again, must be in the radical of $\mathbb{M}_{2}(B)$, and so $\beta w+w u=0$ for $w \in B$. Since $B$ cannot have a right identity, we must have $\beta=0$, and then, since $B$ is semisimple, we see that $u=0$. Thus, we have proved that $\operatorname{rad} A=\{0\}$. 
Next, we claim that $M_{\varphi}^{2}$ has finite codimension in $A$, where $M_{\varphi}$ is the kernel of $\varphi$. Indeed, for each $s, t, u, v \in B$, we have

$$
\left(\begin{array}{ll}
s & u \\
t & v
\end{array}\right)=\left(\begin{array}{ll}
0 & 0 \\
0 & 1
\end{array}\right)\left(\begin{array}{ll}
0 & 0 \\
t & v
\end{array}\right)+\left(\begin{array}{ll}
u & 0 \\
0 & 0
\end{array}\right)\left(\begin{array}{ll}
0 & 1 \\
0 & 0
\end{array}\right)+\left(\begin{array}{ll}
0 & 1 \\
0 & 0
\end{array}\right)\left(\begin{array}{ll}
0 & 0 \\
s & 0
\end{array}\right)
$$

so that $\mathbb{M}_{2}(B) \subset M_{\varphi}^{2}$. In fact, we can check that $M_{\varphi}^{2}=M_{\varphi}$.

Consider $X=\mathbb{C}^{2}$ as a unital Banach $A$-bimodule by defining the module multiplications as $\mathbb{M}_{2}(B) X=X \mathbb{M}_{2}(B)=\{0\}$ and the multiplications by $\mathbb{M}_{2}^{u}$ as

$$
\left(\begin{array}{ll}
\alpha & \beta \\
0 & \gamma
\end{array}\right)\left(\begin{array}{l}
\zeta \\
\eta
\end{array}\right)=\left(\begin{array}{c}
\alpha \zeta+\beta \eta \\
\gamma \eta
\end{array}\right) \text { and }\left(\begin{array}{l}
\zeta \\
\eta
\end{array}\right)\left(\begin{array}{ll}
\alpha & \beta \\
0 & \gamma
\end{array}\right)=\left(\begin{array}{c}
\alpha \zeta \\
\alpha \eta
\end{array}\right)
$$

for $\alpha, \beta, \gamma, \zeta, \eta \in \mathbb{C}$. Then $X$ has exactly one minimal $A$-mod- $A$ submodule, namely

$$
Y=\left\{\left(\begin{array}{l}
\zeta \\
0
\end{array}\right): \zeta \in \mathbb{C}\right\}
$$

We see that $Y_{\lambda}=Y_{\rho}=M_{\varphi}$, so that $Y_{\lambda} Y_{\rho}=M_{\varphi}^{2}$ has codimension 1 in $A$.

Now, since $B^{2}$ has infinite codimension in $B$, there exists a discontinuous linear functional $\lambda$ on $B$ such that $\lambda$ is zero on $B^{2}$. Define a discontinuous linear map $D: A \rightarrow X$ by

$$
D:\left(\begin{array}{cc}
\alpha & \beta \\
0 & \gamma
\end{array}\right) \oplus\left(\begin{array}{cc}
s & u \\
t & v
\end{array}\right) \mapsto\left(\begin{array}{c}
\lambda(s) \\
\lambda(t)
\end{array}\right) .
$$

It can be verified that $D$ is a derivation.

\section{Conclusion}

It remains open to determine conditions that are both necessary and sufficient for a (separable) Banach algebra with finite-dimensional radical to have a unique complete norm. We have seen that the necessary condition (in the unital case) 'for each minimal radical ideal $K$, the ideal $K_{\lambda} K_{\rho}$ has finite codimension' is not sufficient. Our construction is, however, not 'systematic' enough to provide a clue on finding and proving additional necessary conditions.

\section{Acknowledgements}

The author wishes to thank his supervisor, Professor H. Garth Dales, for much advice and support. This work was undertaken while the author was studying for 
a $\mathrm{PhD}$ at the University of Leeds, supported financially by the ORS, the School of Mathematics, and the University of Leeds.

\section{References}

[1] E. A. Behrens, Ring theory, Pure and Applied Mathematics 44, Translated from the German by Clive Reis (Academic Press, New York, 1972).

[2] H. G. Dales, Banach algebras and automatic continuity, London Mathematical Society Monographs. New Series. Vol. 24 (The Clarendon Press Oxford University Press, Oxford, 2000).

[3] H. G. Dales and R. J. Loy, 'Uniqueness of the norm topology for Banach algebras with finitedimensional radical", Proc. London Math. Soc. (3) 74 (1997), 633-661.

[4] H. G. Dales and J. P. McClure, 'Continuity of homomorphisms into certain commutative Banach algebras", Proc. London Math. Soc. (3) 26 (1973), 69-81.

[5] P. G. Dixon, 'Nonseparable Banach algebras whose squares are pathological", J. Funct. Anal. 26 (1977), 190-200.

[6] B. E. Johnson, 'The uniqueness of the (complete) norm topology", Bull. Amer. Math. Soc. 73 (1967), 537-539.

[7] — - "The Wedderburn decomposition of Banach algebras with finite dimensional radical", Amer. J. Math. 90 (1968), 866-876.

[8] B. E. Johnson and A. M. Sinclair, 'Continuity of derivations and a problem of Kaplansky", Amer. J. Math. 90 (1968), 1067-1073.

[9] T. Y. Lam, A first course in noncommutative rings, Graduate Texts in Mathematics 131 (Springer, New York, 1991).

[10] R. J. Loy, 'Multilinear mappings and Banach algebras", J. London Math. Soc. (2) 14 (1976), $423-429$.

[11] _ _ 'The uniqueness of norm problem in Banach algebras with finite-dimensional radical', in: Radical Banach algebras and automatic continuity (Long Beach, Calif., 1981), Lecture Notes in Math. 975 (Springer, Berlin, 1983) pp. 317-327.

[12] V. M. Zinde, "The property of "uniqueness of the norm" for commutative Banach algebras with finite-dimensional radical", Vestnik Moskov. Univ. Ser. I Mat. Mekh. 25 (1970), 3-8.

\section{Department of Pure Mathematics \\ University of Leeds \\ Leeds LS2 9JT \\ UK \\ e-mail: hung@maths.leeds.ac.uk}


